\title{
Asymmetry in acute exacerbation of idiopathic pulmonary fibrosis
}

\author{
Akihiko Sokai ${ }^{1}$, Kiminobu Tanizawa ${ }^{2}$, Tomohiro Handa ${ }^{1}$, Takeshi Kubo ${ }^{3}$, \\ Seishu Hashimoto ${ }^{4}$, Kohei Ikezoe ${ }^{1}$, Yoshinari Nakatsuka', Kensaku Aihara ${ }^{5}$, \\ Yoshio Taguchi ${ }^{4}$, Shigeo Muro ${ }^{1}$, Toru Oga ${ }^{2}$, Sonoko Nagai ${ }^{6}$, Takateru Izumi $^{6}$, \\ Toyohiro Hirai ${ }^{1}$, Kazuo Chin $^{2}$ and Michiaki Mishima ${ }^{1}$
}

Affiliations: ${ }^{1}$ Respiratory Medicine, Graduate School of Medicine, Kyoto University, Kyoto, Japan. ${ }^{2}$ Respiratory Care and Sleep Control Medicine, Graduate School of Medicine, Kyoto University, Kyoto, Japan. ${ }^{3}$ Diagnostic Imaging and Nuclear Medicine, Graduate School of Medicine, Kyoto University, Kyoto, Japan. ${ }^{4}$ Respiratory Medicine, Tenri Hospital, Tenri, Japan. ${ }^{5}$ Respiratory Medicine, Saiseikai Noe Hospital, Osaka, Japan. ${ }^{6}$ Respiratory Medicine, Kyoto Central Clinic/Clinical Research Center, Kyoto, Japan.

Correspondence: Kiminobu Tanizawa, Respiratory Care and Sleep Control Medicine, Graduate School of Medicine, Kyoto University, 54 Kawahara-cho, Shogoin, Sakyo-ku, Kyoto 606-8507, Japan.

E-mail: tanizawadkuhp.kyoto-u.ac.jp

ABSTRACT Acute exacerbation (AE) of idiopathic pulmonary fibrosis (IPF) results in poor survival. The objective of the present study was to elucidate the impact of asymmetrical ground-glass opacity (GGO) and/or consolidation on outcomes in patients with AE-IPF.

The cases of 59 consecutive patients with AE-IPF were retrospectively reviewed. High-resolution computed tomography (HRCT) at diagnosis of an AE was assessed to determine the disease extent and asymmetry. Asymmetrical AE was defined as a right-to-left ratio of GGO and consolidation $\geqslant 2.0$ or $\leqslant 0.5$. The impacts of HRCT indices and other clinical parameters on 180-day mortality were analysed.

The overall 180-day mortality rate was 59.2\%, and asymmetrical $\mathrm{AE}$ was observed in 13 patients (22.0\%). A multivariate analysis revealed that asymmetrical AE was a significant predictor of 180-day mortality (hazard ratio $=0.36, \mathrm{p}=0.047$ ), long-term oxygen therapy before $\mathrm{AE}$ and serum lactate dehydrogenase levels. The 180-day mortality of patients with asymmetrical AE was significantly lower than that of patients with symmetrical AE (asymmetrical AE 30.8\% versus symmetrical AE 68.2\%, $\mathrm{p}=0.03$ ).

An asymmetrical distribution of GGO and/or consolidation is a predictor of survival in patients with AE-IPF.

@ERSpublications

Asymmetrical distribution of GGOs and consolidation could indicate better survival in acute exacerbation of IPF http://ow.ly/EaEr307VTRN

Cite this article as: Sokai A, Tanizawa K, Handa T, et al. Asymmetry in acute exacerbation of idiopathic pulmonary fibrosis. ERJ Open Res 2017; 3: 00036-2016 [https://doi.org/10.1183/23120541.00036-2016].

Received: March 112016 | Accepted after revision: Dec 172016

Support statement: The present study was supported in part by a grant from Japan's Ministry of Health, Labor, and Welfare to the Diffuse Lung Diseases Research Group, the Respiratory Failure Study Group, and the Research Program of Intractable Disease. Other support included a KAKENHI Grant-in-Aid for Young Scientists (B) awarded by the Japan Society for the Promotion of Science and grants from the Japan Intractable Diseases Research Foundation. Funding information for this article has been deposited with the Crossref Funder Registry.

Conflict of interest: Disclosures can be found alongside this article at openres.ersjournals.com

Copyright $\odot$ ERS 2017. This article is open access and distributed under the terms of the Creative Commons Attribution Non-Commercial Licence 4.0. 


\section{Introduction}

Acute exacerbation (AE) of idiopathic pulmonary fibrosis (IPF) is recognised as an acute and fatal episode of respiratory deterioration of unidentifiable cause [1-5]. The mortality of AE of IPF (AE-IPF) is $>50 \%$ [3, 6], and AE profoundly affects the survival of patients with IPF; therefore, defining the clinical manifestations and pathogenesis of AE is crucial for improving the prognosis of IPF.

$\mathrm{AE}$ is characterised by new bilateral ground-glass opacities (GGOs) and/or consolidation superimposed on chronic interstitial pneumonia detected on high-resolution computed tomography (HRCT). HRCT indices, such as disease extent and radiological patterns at the time of AE diagnosis, are significant predictors of mortality in AE-IPF [7-10], acute respiratory distress syndrome (ARDS) [11] and acute interstitial pneumonia (AIP) [12], all of which have similar pathological characteristics of diffuse alveolar damage (DAD) [13]. In addition to the disease extent and the radiological patterns observed on HRCT, the asymmetrical distribution of disease has been analysed in the chronic phase of IPF [14]. Although IPFs are essentially bilateral and are often diffuse, a finding of asymmetry on HRCT could provide new insight into the pathogenesis of IPF and provide clinicians with prognostic information. However, the clinical relevance of an asymmetrical disease distribution has not been investigated in AE-IPF.

Therefore, in the present study, we aimed to elucidate the impact of an asymmetrical distribution of disease on outcomes in patients with AE-IPF. We used the HRCT indices obtained at the time of AE diagnosis to assess the extent and distribution of disease, and we investigated the prognostic value of these HRCT indices and that of other clinical parameters.

\section{Materials and methods}

Study subjects

The medical records of all patients with IPF who required emergent hospitalisation at the Departments of Respiratory Medicine of Kyoto University Hospital and Tenri Hospital, from January 2006 to July 2013, were retrospectively analysed. IPF was diagnosed according to the American Thoracic Society/European Respiratory Society diagnostic criteria for IPF [15]. Patients were excluded if they had active neoplasms or did not undergo HRCT on admission. In total, 59 patients who met the diagnostic criteria for AE were included in the present study. The Kyoto University Hospital Institutional Review Board approved the present study (approval no. E2156/R0637).

\section{Diagnostic criteria for acute exacerbations of idiopathic pulmonary fibrosis}

AE-IPF was diagnosed as follows [3, 4]: 1) either a previous or concurrent diagnosis of IPF; 2) unexplained worsening or development of dyspnoea within 30 days; 3) HRCT with new bilateral GGOs and/or consolidation superimposed on background reticulation or honeycombing; 4) no evidence of pulmonary infection by bronchoalveolar lavage (BAL), endotracheal aspiration or other clinical evaluations; and 5) the exclusion of alternative causes, such as left heart failure, pulmonary embolism and identifiable causes of acute lung injury. Without BAL or endotracheal aspiration, pulmonary infection was excluded only when 1) no purulent sputum was detected, 2) the sputum or blood culture was negative, 3) urinary antigen tests for pneumococcus and Legionella were negative, 4) serum $\beta$-D-glucan levels were normal, 5) serum cytomegalovirus antigen was undetected and 6) HRCT images lacked the predominance of segmental opacities.

\section{Data collection and clinical evaluation}

Clinical information was retrospectively collected from medical records. All of the patients underwent blood testing and surveillance for infection on admission. BAL was performed using the recommended procedure $[16,17]$. Survival at 180 days was assessed as the primary outcome.

\section{High-resolution computed tomography evaluation}

All of the patients underwent chest HRCT before AE treatment was initiated. Three independent observers (A.S., T.K. and K.T.) who were blinded to the patients' clinical information reviewed the HRCT images. The images were assessed for GGOs, consolidation, reticulation (intralobular reticular opacification and interlobular septal thickening) and honeycombing, and emphysema. To measure the disease extent semi-quantitatively, the lung fields on each side were divided into the following five zones: at the level of the origin of the great vessels, the mid-arch of the aorta, the carina, the pulmonary venous confluence, and $10 \mathrm{~mm}$ above the top of the right hemi-diaphragm $[14,18]$. The extents of GGO, consolidation, reticulation and honeycombing, and emphysema were scored to the nearest $10 \%$ in the 10 lung zones; these scores were subsequently averaged to obtain the mean scores. GGOs and consolidation accounting for new lesions were grouped as parenchymal opacities, and reticulation and honeycombing were grouped as fibrotic abnormalities. To assess the asymmetries of GGOs and consolidation, the right-to-left ratio of parenchymal opacities was calculated. When the ratio was $\geqslant 2.0$ or $\leqslant 0.5$, the patient was diagnosed with asymmetrical AE (figure 1 ). The presence or absence of bronchiectasis in parenchymal opacities was also assessed in each case. 
Statistical analyses

The statistical analyses were performed using JMP, version 10.0 (SAS Institute Inc., Cary, NC, USA). All statistical variations in the quantitative data are expressed as the mean \pm SD, and $\mathrm{p}<0.05$ was considered statistically significant. Comparisons between the groups were performed using either Fisher's exact test or the Mann-Whitney $U$ test, as appropriate. To define the prognostic value of
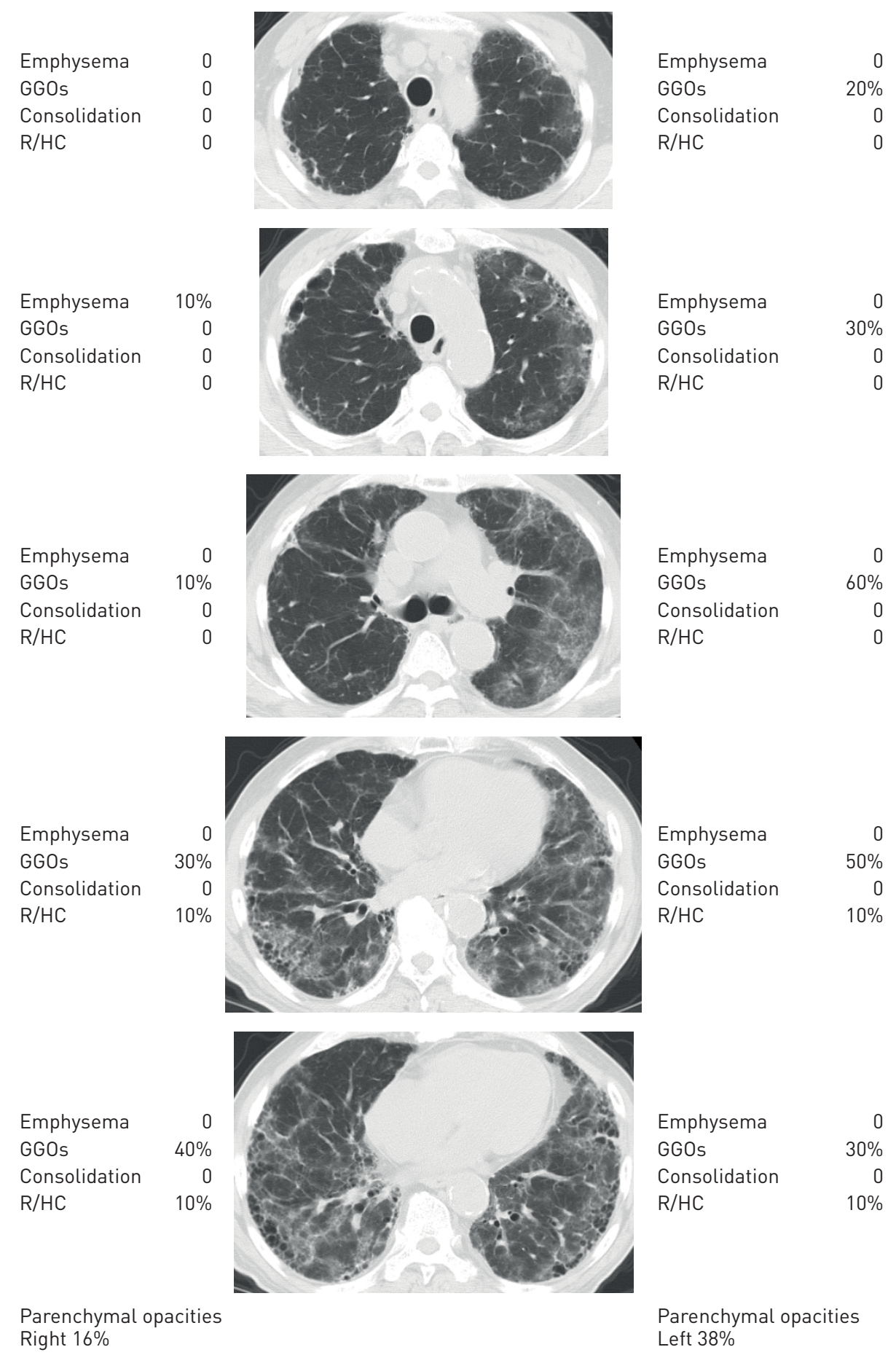

The right-to-left ratio 0.42

FIGURE 1 Evaluation by high-resolution computed tomography (HRCT). The extents of emphysema, ground-glass opacities (GGOs), consolidation, and reticulation and honeycombing (R/HC) were scored to the nearest $10 \%$ in each of the five HRCT slices. The scores of 10 zones were averaged to obtain the mean scores for each lung. The right-to-left ratio of the parenchymal opacities consisting of GGOs and consolidation was calculated. This case, in which the ratio was 0.42 , was an example of asymmetrical acute exacerbation. 


\section{TABLE 1 Patient characteristics and clinical data}

\begin{tabular}{|c|c|}
\hline \multicolumn{2}{|l|}{ Characteristic } \\
\hline Subjects $\mathrm{n}$ & 59 \\
\hline Males & 54 (91.5) \\
\hline Age years & $71.7 \pm 8.2$ \\
\hline Smoking history & $49(86.0)$ \\
\hline FVC before AE \% predicted & $71.0 \pm 21.9$ \\
\hline DLco before AE \% predicted & $37.0 \pm 17.3$ \\
\hline \multicolumn{2}{|l|}{ Treatment before $\mathrm{AE}$} \\
\hline LTOT & 23 (39.0) \\
\hline Corticosteroids & 20 (33.9) \\
\hline Immunosuppressive agents & 10 (16.9) \\
\hline WBC $\mu \mathrm{L}^{-1}$ & $10490 \pm 4150$ \\
\hline $\mathrm{D}$-dimer $\mu \mathrm{g} \cdot \mathrm{mL}^{-1}$ & $5.1 \pm 6.3$ \\
\hline CRP $\mathrm{mg} \cdot \mathrm{dL}^{-1}$ & $6.5 \pm 6.4$ \\
\hline LDH IU.L ${ }^{-1}$ & $380 \pm 226$ \\
\hline $\mathrm{KL}-6 \mathrm{U} \cdot \mathrm{mL}^{-1}$ & $1763 \pm 1278$ \\
\hline$P / F<200$ & $26(47.3)$ \\
\hline BAL performed & $11(18.6)$ \\
\hline \multicolumn{2}{|l|}{ Treatment of $\mathrm{AE}$} \\
\hline High-dose corticosteroids & $59(100)$ \\
\hline Antibiotics & 58 (98.3) \\
\hline Immunosuppressive agents & $30(50.8)$ \\
\hline Noninvasive ventilation & $14(23.7)$ \\
\hline 180-day mortality \% & 59.2 \\
\hline
\end{tabular}

The data are presented as mean \pm SD or $n(\%)$, unless otherwise indicated. FVC: forced vital capacity; AE: acute exacerbation; DLCO: diffusing capacity of the lung for carbon monoxide; LTOT: long-term oxygen therapy: WBC: white blood cell; CRP: C-reactive protein; LDH: lactate dehydrogenase; KL-6: Krebs von den Lungen-6; P/F: the ratio of partial pressure of arterial oxygen to the fraction of inspired oxygen; BAL: bronchoalveolar lavage.

each variable at diagnosis, a survival analysis of the initial 180 days was performed using the Cox proportional hazards model.

Variables were entered into a multivariate analysis model only when they had p-values $\leqslant 0.10$ in the preceding univariate analyses. The 180-day mortality was calculated, and survival curves were generated using the Kaplan-Meier method and were compared between groups using the log-rank test.

\section{Results}

Clinical characteristics

The study included 59 patients with IPF. The clinical data obtained at the time of AE diagnosis and the outcomes are presented in table 1 . The mean age was 71.7 years, and 54 of the patients $(91.5 \%)$ were male. BAL was performed in 11 patients (18.6\%).

\section{High-resolution computed tomography indices at diagnosis of an acute exacerbation}

The HRCT indices at the time of an AE diagnosis are presented in table 2. The mean extent of the parenchymal opacities reflecting the extent of GGOs and consolidation was $40.5 \%$, whereas the mean extent of reticulation and honeycombing was $14.8 \%$. Asymmetrical AE was observed in 13 patients (22.0\%).

\section{TABLE 2 Chest high-resolution computed tomography indices at diagnosis}

The data are presented as mean \pm SD or $n(\%)$. 


\begin{tabular}{|c|c|c|c|c|c|c|}
\hline & \multicolumn{3}{|c|}{ Univariate analysis } & \multicolumn{3}{|c|}{ Multivariate analysis } \\
\hline & HR & $95 \% \mathrm{Cl}$ & p-value & HR & $95 \% \mathrm{Cl}$ & p-value \\
\hline Age & 1.00 & $0.95-1.05$ & 0.96 & & & \\
\hline Male & 1.97 & $0.59-12.2$ & 0.30 & & & \\
\hline Smoking history & 0.44 & $0.20-1.11$ & 0.08 & 0.51 & $0.23-1.31$ & 0.15 \\
\hline LTOT & 2.10 & $1.06-4.25$ & $0.03^{*}$ & 2.34 & $1.04-5.28$ & $0.04^{*}$ \\
\hline FVC before $A E \%$ predicted & 0.99 & $0.97-1.01$ & 0.43 & & & \\
\hline DLco before AE \% predicted & 0.98 & $0.94-1.01$ & 0.16 & & & \\
\hline$P / F<200$ & 0.69 & $0.33-1.40$ & 0.30 & & & \\
\hline CRP & 1.03 & $0.96-1.95$ & 0.36 & & & \\
\hline LDH $10 \mathrm{IU} \cdot \mathrm{L}^{-1}$ per unit & 1.02 & $1.01-1.03$ & $<0.01 *$ & 1.02 & $1.00-1.05$ & $0.02 *$ \\
\hline $\mathrm{KL}-6100 \mathrm{U} \cdot \mathrm{mL}^{-1}$ per unit & 1.02 & $1.00-1.04$ & 0.10 & 0.99 & $0.96-1.02$ & 0.69 \\
\hline Parenchymal opacities & 1.02 & $1.00-1.04$ & 0.09 & 0.99 & $0.96-1.02$ & 0.51 \\
\hline Ground-glass opacities & 1.01 & $0.99-1.03$ & 0.27 & & & \\
\hline Consolidation & 1.02 & $0.98-1.06$ & 0.26 & & & \\
\hline Reticulation/honeycombing & 0.98 & $0.95-1.02$ & 0.31 & & & \\
\hline Emphysema & 1.02 & $0.98-1.05$ & 0.34 & & & \\
\hline Bronchiectasis in parenchymal opacities & 1.19 & $0.60-2.38$ & 0.62 & & & \\
\hline Asymmetrical AE & 0.33 & $0.10-0.85$ & $0.02 *$ & 0.36 & $0.10-0.99$ & $0.047^{*}$ \\
\hline
\end{tabular}

HR: hazard ratio; LTOT: long-term oxygen therapy; FVC: forced vital capacity; AE: acute exacerbation; $D L C O$ : diffusing capacity of the lung for carbon monoxide; P/F: the ratio of partial pressure of arterial oxygen to the fraction of inspired oxygen; CRP: C-reactive protein; LDH: lactate dehydrogenase; KL-6: Krebs von den Lungen-6. *: $p<0.05$.

\section{Therapeutic regimens and outcomes}

Following the diagnosis of AE, almost all of the patients received high-dose corticosteroids and antibiotics simultaneously (table 1). The 180-day mortality was 59.2\%.

\section{The prognostic factors for 180-day mortality}

The results of the univariate and multivariate analyses of the predictors of 180-day mortality are provided in table 3. The univariate analyses revealed that long-term oxygen therapy (LTOT) prior to the diagnosis of $\mathrm{AE}$, level of serum lactate dehydrogenase (LDH) and asymmetrical AE were associated with 180-day mortality. Following the multivariate analysis, LTOT, LDH (10 IU $\cdot \mathrm{L}^{-1}$ per unit) and asymmetrical AE remained significant (LTOT, hazard ratio 2.34, 95\% CI 1.04-5.28, $\mathrm{p}=0.04$; LDH, hazard ratio 1.02, $95 \% \mathrm{CI}$ $1.00-1.05, \mathrm{p}=0.02$; asymmetrical AE, hazard ratio $0.36,95 \%$ CI $0.10-0.99, \mathrm{p}=0.047$ ), whereas the extent of GGOs and consolidation was not a predictor of 180-day mortality. The estimated 180-day mortality of the patients with asymmetrical $\mathrm{AE}$ was significantly lower than that of the patients with symmetrical $\mathrm{AE}$ (asymmetrical AE $30.8 \%$ versus symmetrical AE 68.2\%; $\mathrm{p}=0.03$ ) (figure 2).

\section{Comparison between asymmetrical and symmetrical acute exacerbations}

To define the clinical and radiological features of asymmetrical AE, asymmetrical and symmetrical AEs were compared (table 4). Demographic characteristics, the most recently measured percentage of predicted forced vital capacity (FVC) before the diagnosis of AE, treatment and laboratory data, the ratio of partial pressure of arterial oxygen to the fraction of inspired oxygen, the rates of BAL performance at the time of $\mathrm{AE}$, and the therapeutic regimens following the diagnosis of AE were similar. HRCT performed at the diagnosis of AE revealed that GGOs and parenchymal opacities were less extensive in the asymmetrical $\mathrm{AE}$ group (asymmetrical AE $24.1 \pm 14.4 \%$ and $32.6 \pm 14.5 \%$ versus symmetrical AE $37.0 \pm 14.2 \%$ and $42.7 \pm 14.6 \%$; $\mathrm{p}<0.01$ and 0.04 , respectively).

\section{Discussion}

An asymmetrical distribution of GGOs and consolidation was observed in $22.0 \%$ of patients at the diagnosis of AE-IPF. Asymmetrical AE was a significant predictor of a better outcome, even after adjusting for other possible prognostic factors. Asymmetrical AE was associated with less extensive GGOs, whereas other radiological and clinical features were similar between the patients with symmetrical and asymmetrical AEs.

An asymmetrical disease distribution in interstitial lung diseases has not been addressed, with the exception of a single study of chronic IPF [14]. TCHERAKIAn et al. reported that asymmetrical IPF, which was defined as asymmetrical fibrosis with a 1.5-fold greater extent in one lung than the other, was 


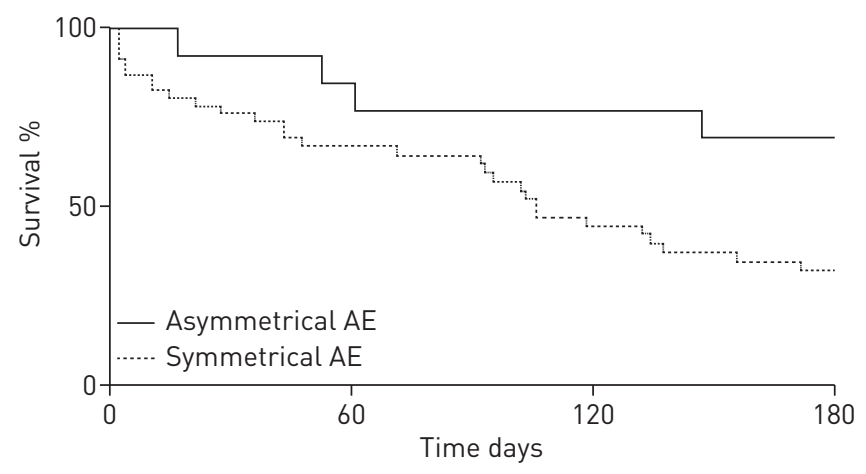

FIGURE 2 A comparison of 180-day survival curves between patients with idiopathic pulmonary fibrosis with asymmetrical acute exacerbation (AE) and those with symmetrical AE.

observed in some patients during the chronic phase [14]. The authors revealed that asymmetrical IPF was associated with gastro-oesophageal reflux disease (GERD) and a higher incidence of AE, suggesting the clinical relevance of asymmetry in interstitial lung diseases. However, asymmetrical distributions on HRCT have not been assessed in AE-IPF, AIP or ARDS. The present study was the first to address asymmetry in AE-IPF and elucidate the clinical implications of asymmetrical AE. Our findings have raised three possible hypotheses regarding the relationship between asymmetrical AE and improved outcomes.

First, asymmetrical AE may represent an earlier phase of AE, before the lesions extend and become both symmetrical and diffuse. Preceding HRCT studies of AE-IPF revealed that more extensive lesions in the late and proliferative stages increased the risk of a worse prognosis [8], highlighting the potential impact of a delay in diagnosis [19]. The better prognoses associated with asymmetrical AE may be related to the earlier detection of $\mathrm{AE}$ during the asymmetrical phase and the subsequent implementation of immediate therapeutic interventions.

Second, asymmetrical AE may be a phenotype that is distinct from symmetrical AE. AKIRA et al. reported that peripheral patterns of AE-IPF did not evolve into diffuse patterns, whereas multifocal patterns represented an early manifestation of a diffuse pattern [7], suggesting the existence of at least two different phenotypes of AE-IPF. Additionally, the histopathology of AE is characterised by DAD and organising pneumonia (OP) superimposed on fibrotic interstitial pneumonia $[20,21]$. OP lesions are generally more localised than DAD; therefore, the asymmetrical distribution of GGOs and consolidation may reflect the predominance of OP lesions. Such different pathological features may affect the disease distribution, responses to treatment and outcomes.

Third, asymmetrical AE may reflect locoregional factors such as GERD and unidentified infections. In asymmetrical IPF, the first AE is virtually unilateral and more frequently occurs in the affected lung [14], suggesting the role of GERD in both chronic fibrosis and AE. Another potential locoregional factor is unidentified infection. Although bilateral infectious deteriorations of IPF were reported to have poor outcomes similar to those of non-infectious $\mathrm{AE}$ [22], unidentified infectious agents may affect the outcome of AE-IPF [3, 23]. We made every effort to exclude infectious causes in the diagnosis of AE in this cohort; however, the possibility of unidentified infection remained. Furthermore, the extent of emphysema in patients with asymmetrical AE was higher than in patients with symmetrical AE. Ventilation-perfusion mismatch due to emphysema may affect the asymmetrical distribution, although the extent of emphysema itself was not associated with mortality.

Previous studies revealed that the presence of a diffuse pattern, lesion extent, HRCT score and centrilobular emphysema on HRCT may be prognostic factors for mortality among patients with AE-IPF and IIPs [7-9]. AKIRA et al. found that in AE-IPF, a poorer prognosis was associated with a diffuse pattern compared with either a peripheral or a multifocal pattern [7]. FUлмото et al. adapted the HRCT scoring system [8], which had been used previously to predict the prognosis in ARDS and AIP to AE-IPF [12, 24], and they demonstrated that HRCT scores and the extent of GGOs with traction bronchiectasis and honeycombing were associated with increased mortality [8]. These preceding studies and ours suggest the prognostic value of HRCT assessment at the time of AE diagnosis, and our results also indicate the need to evaluate the asymmetrical distribution of disease in the acute phase of IPF.

Some limitations of the present study should be mentioned. First, only $18.6 \%$ of the patients underwent $\mathrm{BAL}$ at the time of diagnosis, although the proportion of patients who underwent BAL did not differ according to the clinical group, the presence of asymmetrical AE or patient outcomes. The latest diagnostic criteria for AE-IPF include cases with underlying triggers, such as infection and surgery (triggered AE), as well as those without triggers (idiopathic AE) [25]. While the present study excluded cases with apparent triggers, such as pneumonia, the clinical implications of asymmetrical distribution should be re-evaluated 
TABLE 4 Patient characteristics between asymmetrical and symmetrical acute exacerbation

\begin{tabular}{|c|c|c|c|}
\hline & Asymmetrical AE & Symmetrical AE & p-value \\
\hline Subjects $n$ & 13 & 46 & \\
\hline Age years & $74.2 \pm 8.1$ & $71.0 \pm 8.1$ & 0.21 \\
\hline Male & 10 (76.9) & $44(95.7)$ & 0.07 \\
\hline Smoking history & $3(60.0)$ & 21 (91.3) & 0.14 \\
\hline FVC before $A E \%$ predicted & $70.2 \pm 17.6$ & $71.3 \pm 23.3$ & 0.70 \\
\hline DLCo before AE \% predicted & $34.6 \pm 20.5$ & $38.0 \pm 16.2$ & 0.32 \\
\hline \multicolumn{4}{|l|}{ Treatment before $\mathrm{AE}$} \\
\hline LTOT & $4(30.8)$ & 19 (41.3) & 0.54 \\
\hline Corticosteroids & $2(15.4)$ & $18(39.1)$ & 0.18 \\
\hline Immunosuppressive agents & $0(0.0)$ & 10 (21.7) & 0.10 \\
\hline WBC $\times 10^{3} \cdot \mu \mathrm{L}^{-1}$ & $10120 \pm 4720$ & $10590 \pm 4020$ & 0.43 \\
\hline $\mathrm{D}-$ dimer $\mu \mathrm{g} \cdot \mathrm{mL}^{-1}$ & $4.7 \pm 5.3$ & $5.3 \pm 6.7$ & 1.00 \\
\hline CRP $\mathrm{mg} \cdot \mathrm{dL}^{-1}$ & $5.7 \pm 4.6$ & $6.7 \pm 6.8$ & 0.96 \\
\hline LDH IU:L ${ }^{-1}$ & $347 \pm 88$ & $389 \pm 252$ & 0.82 \\
\hline $\mathrm{KL}-6 \mathrm{U} \cdot \mathrm{mL}^{-1}$ & $1607 \pm 1074$ & $1805 \pm 1335$ & 0.54 \\
\hline$P / F<200$ & $8(61.5)$ & $18(42.9)$ & 0.34 \\
\hline BAL performed & $1(7.7)$ & $10(21.7)$ & 0.43 \\
\hline Parenchymal opacities \% & $32.6 \pm 14.5$ & $42.7 \pm 14.6$ & $0.04^{*}$ \\
\hline Ground-glass opacities $\%$ & $24.1 \pm 14.4$ & $37.0 \pm 14.2$ & $<0.01^{*}$ \\
\hline Consolidation $\%$ & $8.6 \pm 9.2$ & $5.7 \pm 7.0$ & 0.18 \\
\hline Reticulation/honeycombing \% & $16.5 \pm 11.3$ & $14.3 \pm 12.4$ & 0.41 \\
\hline Emphysema \% & $11.7 \pm 13.1$ & $5.7 \pm 9.0$ & 0.07 \\
\hline Bronchiectasis in parenchymal opacities & $6(47.5)$ & $22(47.8)$ & 1.00 \\
\hline \multicolumn{4}{|l|}{ Treatment of $\mathrm{AE}$} \\
\hline High-dose corticosteroids & $13(100)$ & $46(100)$ & - \\
\hline Antibiotics & $13(100)$ & $45(97.8)$ & 1.00 \\
\hline Immunosuppressive agents & $4(30.8)$ & $26(56.5)$ & 0.13 \\
\hline Non-invasive ventilation & $3(23.1)$ & $11(23.9)$ & 1.00 \\
\hline 180-day mortality $\%$ & 30.8 & 68.2 & $0.03^{*}$ \\
\hline \multicolumn{4}{|c|}{$\begin{array}{l}\text { The data are presented as mean } \pm \text { SD or } n(\%) \text {, unless otherwise indicated. AE: acute exacerbation; } \\
\text { FVC: forced vital capacity; DLCO: diffusing capacity of the lung for carbon monoxide; LTOT: long-term } \\
\text { oxygen therapy; WBC: white blood cell; CRP: C-reactive protein; LDH: lactate dehydrogenase; KL-6: Krebs } \\
\text { von den Lungen-6; P/F: the ratio of partial pressure of arterial oxygen to the fraction of inspired oxygen; } \\
\text { BAL: bronchoalveolar lavage. *: } p<0.05 \text {. }\end{array}$} \\
\hline
\end{tabular}

under this more comprehensive concept of AE. Second, we evaluated bronchiectasis in parenchymal opacities in five slices. The prevalence may have been underestimated because traction bronchiolectasis or bronchiectasis with GGO or consolidation was originally assessed for zones of each lung $[8,12]$.

Despite these limitations, we have demonstrated the clinical relevance of an asymmetrical distribution for the diagnosis of AE-IPF. Among the HRCT indices recorded at the time of diagnosis, asymmetrical AE predicts better outcomes. Attention should be paid to both asymmetry and disease extent on HRCT because these factors can provide additional clues for a better understanding of AE-IPF and a more precise prediction of its course.

\section{References}

1 Park IN, Kim DS, Shim TS, et al. Acute exacerbation of interstitial pneumonia other than idiopathic pulmonary fibrosis. Chest 2007; 132: 214-220.

2 Suda T, Kaida Y, Nakamura Y, et al. Acute exacerbation of interstitial pneumonia associated with collagen vascular diseases. Respir Med 2009; 103: 846-853.

3 Collard HR, Moore BB, Flaherty KR, et al. Acute exacerbations of idiopathic pulmonary fibrosis. Am J Respir Crit Care Med 2007; 176: 636-643.

4 Tachikawa $\mathrm{R}$, Tomii $\mathrm{K}$, Ueda $\mathrm{H}$, et al. Clinical features and outcome of acute exacerbation of interstitial pneumonia: collagen vascular diseases-related versus idiopathic. Respiration 2012; 83: 20-27.

5 Papanikolaou IC, Drakopanagiotakis F, Polychronopoulos VS. Acute exacerbations of interstitial lung diseases. Curr Opin Pulm Med 2010; 16: 480-486.

6 Antoniou KM, Wells AU. Acute exacerbations of idiopathic pulmonary fibrosis. Respiration 2013; 86: 265-274.

7 Akira M, Kozuka T, Yamamoto S, et al. Computed tomography findings in acute exacerbation of idiopathic pulmonary fibrosis. Am J Respir Crit Care Med 2008; 178: 372-378.

8 Fujimoto K, Taniguchi H, Johkoh T, et al. Acute exacerbation of idiopathic pulmonary fibrosis: high-resolution CT scores predict mortality. Eur Radiol 2012; 22: 83-92. 
9 Usui Y, Kaga A, Sakai F, et al. A cohort study of mortality predictors in patients with acute exacerbation of chronic fibrosing interstitial pneumonia. BMJ Open 2013; 3.

10 Bhatti H, Girdhar A, Usman F, et al. Approach to acute exacerbation of idiopathic pulmonary fibrosis. Ann Thorac Med 2013; 8: 71-77.

11 Ichikado K, Muranaka H, Gushima Y, et al. Fibroproliferative changes on high-resolution CT in the acute respiratory distress syndrome predict mortality and ventilator dependency: a prospective observational cohort study. BMJ Open 2012; 2: e000545.

12 Ichikado K, Suga M, Müller NL, et al. Acute interstitial pneumonia: comparison of high-resolution computed tomography findings between survivors and nonsurvivors. Am J Respir Crit Care Med 2002; 165: 1551-1556.

13 Kaarteenaho R, Kinnula VL. Diffuse alveolar damage: a common phenomenon in progressive interstitial lung disorders. Pulm Med 2011; 2011: 531302.

14 Tcherakian C, Cottin V, Brillet PY, et al. Progression of idiopathic pulmonary fibrosis: lessons from asymmetrical disease. Thorax 2011; 66: 226-231.

15 American Thoracic Society/European Respiratory Society. American Thoracic Society/European Respiratory Society International Multidisciplinary Consensus Classification of the Idiopathic Interstitial Pneumonias. This joint statement of the American Thoracic Society (ATS), and the European Respiratory Society (ERS) was adopted by the ATS board of directors, June 2001 and by the ERS Executive Committee, June 2001. Am J Respir Crit Care Med 2002; 165: 277-304.

16 Meyer KC, Raghu G, Baughman RP, et al. An official American Thoracic Society Clinical Practice Guideline: the clinical utility of bronchoalveolar lavage cellular analysis in interstitial lung disease. Am J Respir Crit Care Med 2012; 185: 1004-1014.

17 Klech H, Pohl W (eds). Technical recommendations and guidelines for bronchoalveolar lavage (BAL). Report of the European Society of Pneumology Task Group. Eur Respir J 1989; 2: 561-585.

18 Wells AU, Rubens MB, du Bois RM, et al. Serial CT in fibrosing alveolitis: prognostic significance of the initial pattern. AJR Am J Roentgenol 1993; 161: 1159-1165.

19 Simon-Blancal V, Freynet O, Nunes H, et al. Acute exacerbation of idiopathic pulmonary fibrosis: outcome and prognostic factors. Respiration 2012; 83: 28-35.

20 Churg A, Wright JL, Tazelaar HD. Acute exacerbations of fibrotic interstitial lung disease. Histopathology 2011; 58: 525-530.

21 Churg A, Müller NL, Silva CI, et al. Acute exacerbation (acute lung injury of unknown cause) in UIP and other forms of fibrotic interstitial pneumonias. Am J Surg Pathol 2007; 31: 277-284.

22 Song JW, Hong SB, Lim CM, et al. Acute exacerbation of idiopathic pulmonary fibrosis: incidence, risk factors and outcome. Eur Respir J 2011; 37: 356-363.

23 Parambil JG, Myers JL, Ryu JH. Histopathologic features and outcome of patients with acute exacerbation of idiopathic pulmonary fibrosis undergoing surgical lung biopsy. Chest 2005; 128: 3310-3315.

24 Ichikado K, Suga M, Muranaka H, et al. Prediction of prognosis for acute respiratory distress syndrome with thin-section CT: validation in 44 cases. Radiology 2006; 238: 321-329.

25 Collard HR, Ryerson CJ, Corte TJ, et al. Acute exacerbation of idiopathic pulmonary fibrosis. An International Working Group Report. Am J Respir Crit Care Med 2016; 194: 265-275. 